\title{
C. NORBANO (COS. 83 A.C.) EN SICILIA. UNA NOTA
}

\author{
LUIS AMELA VALVERDE \\ Grupo CEIPAC \\ Universidad de Barcelona \\ amelavalverde@gmal.com \\ ORCID: 0000-0002-2485-4815
}

\section{RESUMEN}

C. Norbano (cos. 83 a.C.) fue uno de los políticos más destacados de la factio popularis de finales del siglo II e inicios del siglo I a.C. En el siguiente trabajo pretendemos abordar el gobierno de este personaje en la isla de Sicilia durante la primera mitad de la década de los años 80s del siglo I a.C. Abordamos la cronología de su gobernación, su mención en un miliario, su actividad militar durante estos años y su mención en un epígrafe de Rhegium.

PALABRAS CLAVE: Norbano, Sicilia, Guerra de los Aliados, Primera Guerra Civil, Rhegium.

\section{NORBANUS (COS. 83 BC) IN SICILY. A NOTE}

\section{ABSTARCT}

C. Norbanus (cos. $83 \mathrm{BC}$ ) was one of the most prominent politicians of the factio popularis of the late 2 nd century and early 1 st century BC. In the following work we intend to address the government of this personage on the island of Sicily during the first half of the decade of the 80s of the 1st century BC. We deal with the chronology of his government, the mention of him in a milestone, his military activity during these years and the mention of him in an epigraph of Rhegium.

KEYWORDS: Norbanus, Sicily, Social War, First Civil War, Rhegium.

C. Norbano (cos. 83 a.C.), un político con un nombre extraño, se convirtió en el primer miembro de su familia en obtener el consulado (Evans 1987: 121). Se le ha considerado un novus homo, posiblemente un nuevo ciudadano, y miembro de una factio creada por C. Mario (cos. I 107 a.C.), cuyos miembros fueron promovidos a la más alta magistratura del estado a través de su auctoritas (Badian 1962: 60, 1983: 156; Gruen 1968: 164; Wiseman 1971: 17 y 245; Di Giacomo 2010: 154). Pero Norbano fue más bien, probablemente, un miembro de una familia senatorial menor (que quizás haya llegado recientemente a esta posición) (Evans 1987: 122). En el presente trabajo pretendemos tratar uno de los aspectos menos conocidos de su biografía: su estancia como gobernador de Sicilia durante la primera mitad de la década de los años 80s a.C., un momento de notable inestabilidad en Italia. 


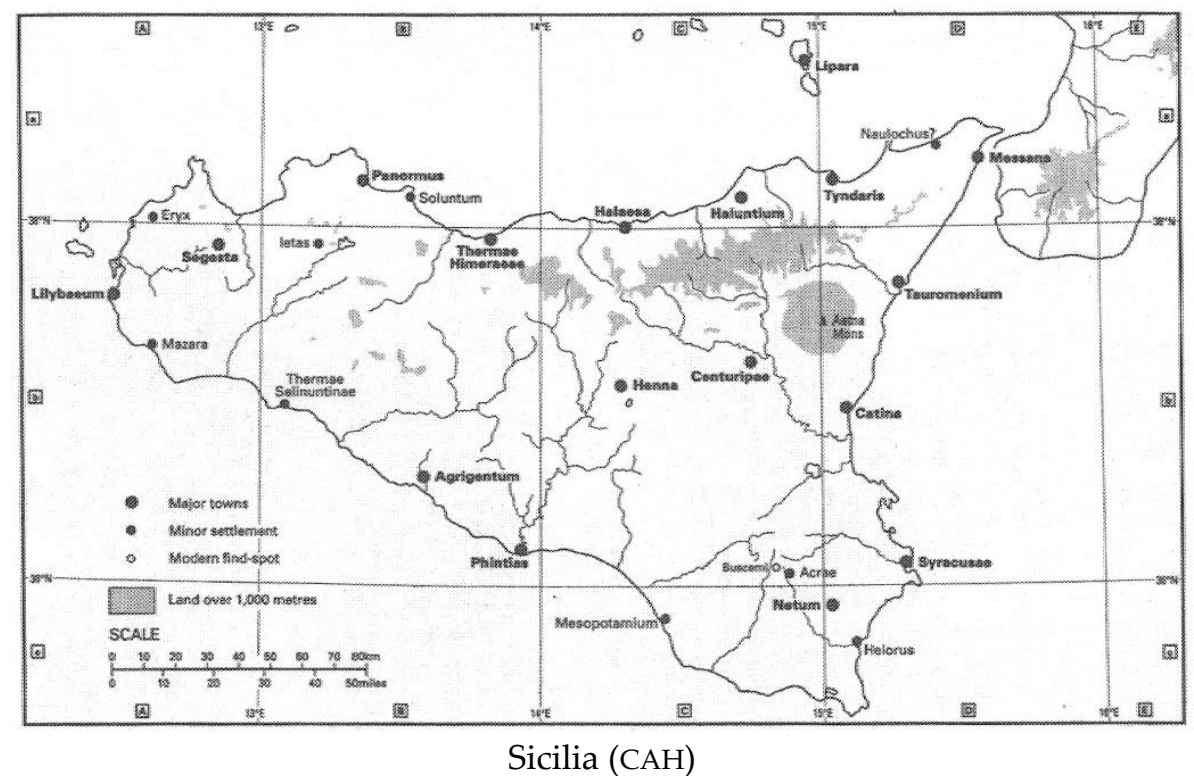

\section{Cronología}

Uno de los puntos más importantes de la carrera de Norbano fue su cargo de gobernador de la provincia de Sicilia como pretor. ${ }^{1}$ De esta forma, se le ha dado como praetor Siciliae ca. en el año 91 a.C. (Díaz Fernández 2015: 308, 2017: 82 y 90), en el año 90 a.C. (Prag 2007b: 252, Di Giacomo 2010: 155, Díaz Ariño 2015: 81), en el año 89 a.C. (Campagna 2007: 115), en el año 88 a.C. (Evans 1987: 264, Konrad 1997: 54, Uggeri 2007: 232), en el año 88/87 a.C. (Manganaro 1972: 453) o en el año 87 a.C. (Münzer 1932: 285, Holm 1965: 626, Evans 1995: 37, Micciché 2020: 452); en principio, se le reconocía un gobierno provincial durante los años 88-87 a.C. (Jashemki 1950: 116, Walton 1967: 197 n. 3, Wiseman 1971: 245, Matsubara 1999: 122), Más recientemente, J. R. W. Prag ha señalado que Norbano pudo ser pretor en el año 90 a.C. (en interrogante) y propretor durante los años 89-85 a.C. (en interrogante la última fecha) (Prag 2007c: 302, Pina Polo y Díaz Fernández 2019: 212), aunque en otro lugar ya lo hace pretor en Sicilia desde el año 90 a.C. (Prag 2007b: 253); en su momento, E. Badian pensó que Norbano fue gobernador ca. los años 90 a 87 a.C., siendo pretor en el mismo año (Badian 1964: 85 y 96). Así mismo, A. Díaz Fernández le ha otorgado las fechas de los años ¿91-89? a.C. (Díaz Fernández 2015: 308, 2017: 90), mientras que G. Manganaro lo hace durante los años 90-87 a.C. (Manganaro 2012: 73), C. Soriaci los años 90-88 a.C. (Soriaci 2016: 173), L. Campagna los años 89-87 a.C. (Campagna 2007: 115), y J. Andreau al menos para los años 90-89 a.C. y quizás más tiempo (Andreau 2007: 83). En definitiva, podemos decir que el gobierno provincial de Norbano tuvo lugar durante el decenio de los años 80s a.C. (Wilson 2017: 98), en su primera mitad.

En su día, T. R. S. Broughton planteó (en interrogante) si Norbano fue pretor en el año 88 a.C. como gobernador de Sicilia, que no tuvo problemas con la

\footnotetext{
${ }^{1}$ Fuentes en Broughton 1952: 41 y 48.
} 
Guerra de los Aliados (91-89/87 a.C.) que se desarrollaba en Italia (Cic. 2Verr. 2, 3, 117a; 2, 5, 8. Diod. 37, 2, 13-14) (Broughton 1952: 41). En cualquier caso, no estaría in summo otio, como afirmó Cicerón (Cic. Verr. 2, 5, 8), si realmente defendió Rhegium de los itálicos rebeldes (Manganaro 1989: 179), vid infra. Norbano mantendría su puesto durante el año 87 a.C. (Broughton 1952: 45 n. 2 y 48).

Posteriormente, T. R. S. Broughton sitúa la pretura de Norbano en el año 89 a.C. y su propretura en los años 88-87 a.C., puesto que, de acuerdo a Diodoro (Diod. 37, 2, 13) y Cicerón (Cic. 2Verr. 2, 3, 117; 2, 5, 8), mantuvo el mando durante la Guerra de los Aliados (Broughton 1986: 149, Manganaro 1989: 178), e hipotetiza si pudo mantenerse aquí durante los consulados de L. Cornelio Cinna (Broughton 1986: 149), es decir, durante los años 87-84 a.C. Sea como fuere, T. C. Brennan señala que los cinco pretores/propretores que tenían mando provincial entre los años 92 y 88 a.C. fueron todos continuamente prorrogados, entre ellos Norbano, debido a la emergencia derivada de la Guerra de los Aliados (Brennan 2000: 707-708).

Como indica J. R. W. Prag, la fecha exacta del gobierno de Norbano en Sicilia es incierta, y es posible que pudiera extenderse entre el inicio de la Guerra de los Aliados (91 a.C.) hasta su vuelta a Roma para el consulado del año 83 a.C. (Prag 2007c: 302); posiblemente regresó a la Ciudad Eterna en el año 85 a.C., ya que en el año 84 a.C. fue candidato al consulado, ganando las elecciones (Andreau 2007: 83).

El sucesor de Norbano pudo haber sido C. Perperna (pr. 85? a.C.) (Dio Cass. 38/39, 14, 1. Plut. Pomp. 10, 1. Cf. Val. Max. 6, 2, 8. Vell. Pat. 2, 30, 1), como manifiesta, p.e., J. R. W. Prag, que podía haber estado al frente de la isla desde una fecha tan temprana como inicios del año 85 a.C. (Brennan 2000: 481, 588 y 708; Prag 2007c: 303; Díaz Fernández 2015: 310, 2017: 90; Micciché 2020: 454), una idea que apoya S. Pittia, quien atribuye a éste la pretura en Sicilia ca. el año 85 o 84 a.C. (Pittia 2012: 204), mientras que A. Díaz Fernández ofrece las fechas de los años ¿85?-82 a.C. (Díaz Fernández 2015: 310). De ser así, habría que suponer que Perperna reemplazaría a Norbano al frente de la provincia. ${ }^{2}$

\footnotetext{
${ }^{2}$ Badian 1984: 86 manifestó que Perperna podría o no haber sido el sucesor de Norbano, pero, a tenor de la documentación disponible, entre ellos, si lo hubiese, habría únicamente otro gobernador. De esta manera, Díaz Fernández 2015: 310, 2017: 90 considera que entre ambos podría encontrarse Cn. Aufidio T. f. (IG XIV $612=$ I.Rhegion $1=$ SIG $\left.^{3} 715\right)$, que podría haber sido gobernador de Sicilia durante los años i89-88? a.C., pero tradicionalmente este personaje se ubica a finales del siglo II a.C., como Broughton, 1986: 28, de tal manera que Brennan 2000: 921 n. 401; Díaz Fernández 2017: 82 fecha el gobierno de este personaje a finales del siglo II a.C. o a principios del siglo I a.C., de forma en que en cierta manera se contradice a sí mismo.
} 


\section{El MiLIARIO DE SiRACUSA}

Uno de los documentos que muestran la presencia de Norbano en Sicilia es una inscripción de Castello Eurialo, en Syracusae, uno de los pocos testimonios epigráficos de la red viaria romana en la isla (Manganaro 1989: 180), ${ }^{3}$ procedente de la vía Syracusae-Agrigentum, una reforma (Di Paola 1999: 462, Díaz Ariño 2015: 81), posiblemente con fines militares (Di Giacomo 2010: 155): C(aius) Nor[banus f(ilius) - n(epos) Balbus] / anno [extremo praeturae, / Q(uintus) A]nic[ius? --- / quaestor pro pr(aetore) --- / [uias in]cl[inatas et angustas a] / Syracuss[is ad Acras uorsus], / praeter[missis inuiis semitis] / et ab Ac[ris ad Agrigentum] / vorsus, a[diectis pontibus], / refe[cerunt latiores?] (AE 1989 342a = CIL I 2951). ${ }^{5}$ Esta inscripción se ha fechado, en consonancia con la cronología que se ha otorgado al gobierno de Norbano, ca. los años 90/85 a.C. (Wilson 2013a: 487).

La línea segunda de la inscripción implica que Norbano estuvo activo más de un año en la isla (Manganaro 1989: 179); de aquí, las fechas que antes hemos analizado. Este epígrafe refleja el interés en la defensa estratégica de Sicilia por parte de Roma ante los rebeldes itálicos, al iniciarse la construcción de esta vía al inicio de la Guerra de los Aliados (Manganaro 1989: 180), al menos, en cuanto a los testimonios que tenemos en la actualidad. Esta política continuará durante la subsiguiente Guerra Civil, cuando Cn. Pompeyo Magno (cos. I 70 a.C.) construyó la via Pompeia (Cf. Cic. 2Verr. 2, 5, 66), que comunicaba Messana con Syracusae, trayecto que curiosamente hoy día se llama «Consolare Pompea», durante el año 82 a.C., cuyo fin último sería garantizar el aprovisionamiento de trigo para Roma (Sirena 2007: 101, 2011: 19; Uggeri 2007: 232-233; Soraci 2016: 84; Amela 2020: 240).

En la inscripción aparece la presencia de otro personaje, Q. Anicius L. f. ${ }^{6}$ (Gallus o Balbus [Broughton 1952: 529]), ${ }^{7}$ pero la reconstrucción de su nombre no es ni mucho menos segura, por no decir la posibilidad de que éste fuese quaestor pro praetore (Prag 2007c: 303). Sea como fuere, recientemente se ha defendido ciertamente que este individuo fue cuestor de Norbano ca. el año 86 a.C. (Pina Polo y Díaz Fernández 2019: 212) y, en cualquier caso, el responsable de la reforma o reparación de la citada vía (Brennan 2000: 836 n. 32).

\footnotetext{
${ }^{3}$ Sólo se conoce otro miliario de época republicana procedente de Sicilia (AE $1957172=$ CIL I $2877=$ ILLRP 1277).

${ }^{4}$ Una lectura alternative es: ab Ac[ris ad Lilybaeum].

${ }^{5}$ Díaz Ariño 2015: 81 piensa que esta inscripción, por su estado fragmentario, no puede considerarse como un miliario.

${ }^{6}$ Broughton 1952: 23 recuerda a un Q. Anicius Lf. [Gallus[ como Xvir agris dandis assignandis (CIL x 44) de una comisión de M. Licio Druso (tr. pl. 91 a.C.).

${ }^{7}$ Segín Manganaro 1989: 179 sería el cuestor de Norbano que pudo ejercer algunas funciones de pro praetore provinciae Siciliae, y que pudo actuar como gobernador de Sicilia entre Norbano y Perperna.
} 


\section{SU ACTIVIDAD MILITAR}

Una importante actividad militar fue llevada a cabo por Norbano ca. los años 88$87^{8}$ a.C. (Salmon 1967: 369, Paoletti 1994: 506, Manganaro 1998: 179, Pittia 2012: 203, Dart 2014: 200), posiblemente en este último año (Badian 1964: 85, Walton 1967: 197, Matsubara 1998: 99, Brennan 2000: 481, Rodríguez González 2005: 547, Amela 2007: 170, Di Giacomo 2010: 156 y 163 n. 65, Kendall 2013: 588). ${ }^{9}$ Diodoro, al escribir sobre la Guerra de los Aliados, indicó que los Lucanos aprovecharon la coyuntura de que L. Cornelio Sila (cos. I 88 a.C.) había partido con su ejército a Asia con el objetivo de hacer la guerra contra Mitrídates VI del Ponto (120-63 a.C.), y que Roma se encontraba distraída por grandes y sangrientos disturbios intestinos (Diod. 37, 2, 14), para efectuar operaciones militares de cierta envergadura. La intención de los Lucanos era difundir la alarma y el desaliento en todo el Bruttium, una región muy apropiada para operaciones de guerrilla (Salmon 1967: 369), y, si era posible, invadir la isla de Sicilia (Amela 2007: 170, Pittia 2012: 204). Al frente de los Lucanos se encontraban M. Lamponio, Ti. Clepicio y un tal Pompeyo $^{10}$ (probablemente C. Papio Mutilo) (Salmon 1967: 369, Rodríguez González 2005: 547 y 620, Kendall 2013: 408), «los generales del resto itálico» (Diod. 37, 2, 13).

La única acción conocida de los Lucanos fue sitiar la localidad de Isiae (Walton 1967: 197, Kendall 2013: 408, Dart 2014: 199) o Tisiae (Merimée 1841: 215 n. 1, Salmon 1967: 370, Rodríguez González 2005: 620), fuertemente fortificada, en cuyo asedio persistieron mucho tiempo (Diod. 37, 2, 13). ${ }^{11}$ Al no poder capturarla, los Lucanos dejaron parte de su ejército ocupado en la continuación del sitio, mientras que con el resto de sus fuerzas (sin poder cuantificarlas de modo alguno) intentaron apoderarse de Rhegium (Diod. 37, 2, 14) (el trampolín para cualquier intento sobre Sicilia desde Italia), con la esperanza de que si caía en sus manos pudieran fácilmente transportar sus tropas a esta isla y lograr someterla (Diod. 37, 2, 14) (Salmon 1967: 369, Amela 2007: 171, Kendall 2013: 408).

Este último plan se vio frustrado por Norbano. ${ }^{12}$ Éste, al ser informado de la situación, actuó con prontitud, y «rescató» a los habitantes de Rhegium (Diod. $37,2,14$ ), a la que los Lucanos habían puesto sitio; posiblemente la guarnición (si había alguna) y los habitantes de la plaza resistieron el tiempo suficiente hasta la llegada de la fuerza de socorro (Rodríguez González 2005: 547). Se desconoce si su sola llegada hizo desistir a éstos de su propósito o tuvo que enfrentarse a ellos

\footnotetext{
${ }^{8}$ De manera más concreta, a finales del año 88 a.C. o a principios del año 87 a.C.

${ }_{9}$ Por el contrario, Merimée 1841: 215 n. 1 señaló el año 88 a.C.

${ }^{10}$ Los nombres de estos personajes parece ser que se encuentran corruptos.

${ }^{11}$ Sobre su identificación, vid: C. Turano, «Taisia», Klearchos 13 (1971), 24-26.

${ }^{12} \mathrm{O} \varrho \beta \alpha$ vó la única fuente que transmite esta información, Diodoro.
} 
militarmente. ${ }^{13}$ Sea como fuere, Sicilia se vio libre de la Guerra de los Aliados (Soraci 2016: 83). ${ }^{14}$

En cualquier caso, por estos años se han podido detectar un total de tres tesoros monetarios, que denotan peligro o amenaza de éste (¿piratería?) para la isla: Siracusa 1963 (RRCH 233) (88 a.C.) y Pantelaria (Cossura) (RRCH 243) (85 a.C.) (Manganaro 2012: 77-81), aunque quizás este último sería algo posterior a la moneda que cierra la ocultación, más concretamente con la presencia de Pompeyo en el año 82 a.C. (Manganaro 2012: 82, Amela 2020: 233). Poco más puede añadirse.

Si los Lucanos hubieran tenido éxito en su plan, los Romanos podrían haber tenido otra guerra en sus manos en un país del que habían obtenido una gran ayuda en su contienda con los itálicos rebeldes. Más aún, si Mitrídates vi no se hubiera entretenido en imponerse a la flota de los Rodios, podría haber enviado tropas al sur de Italia en apoyo de los rebeldes, lo que sin duda habría supuesto un gran perjuicio para Roma (Long 1866: 231).

Para H. Last y R. Gardner, con el fracaso de los Lucanos ante Rhegium, cayó el telón de la Guerra de los Aliados (Last y Gardner 1932: 200). Pero, todavía, los Samnitas tenían aún que jugar un papel en la escena política romana propiciada por la Guerra Civil. Sea como fuere, este incidente muestra que los rebeldes itálicos todavía tenían fuerzas suficientes como para atacar ciudades romanas y no estaban completamente derrotados (Sampson 2013: 71).

Cicerón señala a este respecto que «... cum bello sociorum tota Italia arderet, homo non acerrimus nec fortissimus, C. Norbanus, in summo otio fuit; perfacile enim sese Sicilia iam tuebatur, ut ne quod ex ipsa bellum posset existere» (Cic. Verr. 2, 5, 8). De esta manera, durante la Guerra de los Aliados, «un hombre ni muy enérgico ni muy valiente, Norbano, permaneció en la más completa tranquilidad, pues Sicilia se protegía muy fácilmente por sí sola, de forma que no podía surgir ninguna guerra de ella misma». ${ }^{15}$ Palabras taimadas por no decir falsas, que hay que insertar en el famoso proceso contra C. Verres (pr. 74 a.C.), pues Diodoro indica que Norbano, precisamente como precaución ante una posible invasión, había acumulado tropas y recursos (Diod. 37, 2, 14) (Brennan 2000: 836 n. 32, Prag 2007a: 78); igualmente, se ha indicado que esta opinión podría deberse a una

\footnotetext{
${ }^{13}$ Kendall 2013: 409 menciona que Norbano reclutó un enorme ejército y marina, pero no existe evidencia alguna del tamaño de la fuerza del gobernador de Sicilia, y más bien debió ser modesta, En el mismo sentido escribe Rodríguez González 2005: 547, quien piensa que Norbano acudió a Rhegium «con un gran ejército (no cuantificado)».

${ }^{14}$ Holm 1965: 222, sin aclararlo, menciona que si los aliados itálicos hubieran puesto un pie en la isla de Sicilia, los habitantes de Messana quizás les hubieran ayudado, debido posiblemente a que éstos eran de origen también itálico (los mamertinos, «hijos de Marte», exactamente de Mamers, dios osco de la guerra), de Campania, que se habían establecido en la ciudad antes del estallido de la Primera Guerra Púnica (264-248 a.C.) y que, de hecho, dieron origen a este conflicto bélico.

${ }^{15}$ La única otra actuación de Norbano en Sicilia es al de los «'Diezmos adjudicados de Cayo Norbano correspondientes a la zona de Leontinos'» (Cic. Verr. 3, 49, 117).
} 
década de propaganda hostil tras la victoria de Sila (Badian 1964: 85). Ciertamente, Sicilia no se vio perjudicada de manera directa por la Guerra de los Aliados, pero sí contribuyó de otra forma (Jenison 1919: 58).

Cicerón se contradice en parte a sí mismo (aunque lo intenta disimular, evidentemente), cuando manifestó: Itaque ille M. Cato Sapiens cellam penariam rei publicae nostrae, nutricem plebis Romanae Siciliam nominabat. Nos vero experti sumus Italico maximo difficillimoque bello Siciliam nobis non pro penaria cella, sed pro aerario illo maiorum vetere ac referto fuisse; nam sine ullo sumptu nostro coriis tunicis frumentoque suppeditando maximos exercitus nostros vestivit, aluit, armavit (Cic. Verr. 2, 2, 5). De esta forma, durante la Guerra de los Aliados, Sicilia se convirtió en una despensa para Roma, en la que, sin ningún dispendio, "por nuestro lado», suministró «cueros, túnicas y trigo vistió, alimentó y armó a nuestros grandes ejércitos». Así pues, Norbano tenía su disposición suministros y tropas, aunque ya es otro cantar so éstos fueron dados libremente o mediante requisa (Barlow 1980: 206).

Desconocemos el número y la disposición de las fuerzas de las que disponía Norbano (Prag 2007a: 78), pero existe una probable referencia en un fragmento de Salustio (P.Ryl. 473.1) a la utilización de fuerzas locales por un cuestor (innominado, que es de suponer que fuera de Norbano) en la vecindad de Eryx, ca. el año 87 a.C., al oponerse a Mario y sus compañeros, que habían desembarcado a por agua, matando a dieciséis de ellos (cf. Plut. Mar. 40, 2-3). Existían dos cuestores en Sicilia, uno ubicado en Syracusae y otro en Lilybaeum (Díaz Fernández 2015: 122-123), lo que da idea de la importancia de la isla para Roma, en especial en el plano fiscal; posiblemente se trató del cuestor de Lilybaeum (Carney 1961: 111, Brennan 2000: 836 n. 32). Ciertamente, es un tanto sorprendente que Norbano, que debía ser el gobernador en este momento (Sampson 2013: 71, Soraci 2016: 83), un miembro destacado de la factio de Mario, actuase de esta forma (Carney 1961: 111), aunque es posible que el cuestor interviniera por su cuenta, siguiendo órdenes directas de Roma (Carney 1961: 112). Si tuviéramos el nombre de este anónimo personaje, quizás podríamos arrojar un halo de luz a este curioso como misterioso asunto.

Dadas las circunstancias, parece poco probable que Norbano tuviera a su disposición algo más que levas locales (Prag 2007a: 78, Dubouloz y Pittia 2009: 99). No parece existir en Sicilia guarniciones estables de soldados romanos (Prag 2007a: 74), sino que, en caso de problemas, se procedía a utilizar auxilia externa, es decir, unidades formadas por elementos no italianos que sirven en el ejército romano, que ya se encuentran atestiguadas durante la Segunda Guerra Servil (104-100 a.C.): Mauretanos en el año 104 a.C. (Diod. Sic. 36, 5, 4) y Bitinios, Tesalios y Acarnanianos en el año 103 a.C. (Diod. Sic. 36, 8. 1) (Prag 2007a: 74, Dubouloz y Pittia 2009: 98-99). Además, un fragmento de Salustio ya comentado menciona la presencia de soldados africanos en Sicilia (P. Ryl. 473, 1). La presencia de tales unidades parece confirmar la renuencia romana a contratar legionarios o, para decirlo de otra manera, la voluntad de utilizar otro tipo de tropas, como latinos e italianos (Prag 2007a: 75). 
Curiosamente, en Eryx, la sede del cuestor de la Sicilia occidental, se conserva dos inscripciones (CIL I $843=$ CIL X 7258 = ILLRP 843 e IG XIV 282), en la que parece atestiguarse la existencia de una guarnición militar (Prag 2010: 103 n. 7), que estaría compuesta de levas locales (Konrad 1997: 52). También el gobernador de la isla debía disponer de naves, ya que tenemos la noticia de que los barcos de Sicilia fueron a reforzar a la flota popular en el año 85 a.C. (App. BCiv. 1, 76), ${ }^{16}$ aunque se desconoce si fueron enviados por Norbano o por Perperna, y, por supuesto, si eran romanos o de los aliados sicilianos (Prag 2007a: $80)$, circunstancia esta última más probable.

\section{LA INSCRIPCIÓN DE RHEGIUM}

Ha de señalarse que existe una inscripción proveniente de Rhegium (I.Rhegion 4 $=$ SEG I $418=$ SEG XL $854=$ Suppl.It. V 11) dedicada a un C. Norbanus C. f., que puede tratarse de nuestro personaje (Manganaro 1989: 179, Paoletti 1994: 506, Díaz Fernández: 2015: 309, 2017: 82) o de su hijo homónimo (Putorti 1927: 6, Münzer 1932: 234-235, Prag 2007c: 302, Soraci 2016: 83), quien fue triumvir monetalis en el año 83 a.C. (RRC 357/1a-b) (Crawford 1974: 372). A este último respecto, el reverso de los denarios que el hijo del gobernador Norbano acuñó muestran los símbolos del imperium (en el caso de RRC 357/1a tanto en el mar como en tierra) y felicitas, bastante apropiados en una emisión de la factio popular de esta fecha (Crawford 1974: 372), pero, igualmente, quizás sea una alusión al levantamiento del sitio de Rhegium que hemos comentado por parte de su padre como gobernador de Sicilia (Putorti 1927: 6, Mommsen 1870: 453 n. 2, Babelon 1886: 258-259, Grueber 1910: 347 n. 1).

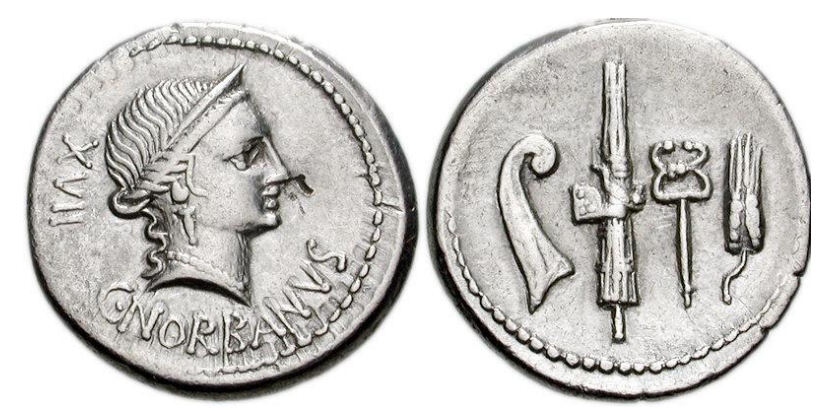

Denario RRC 357/1a de C. Norbano. En el anverso se representa a la diosa Venus y en el reverso una proa, las fasces, un caduceo y una espiga de trigo.

La mencionada inscripción de Rhegium es una base paralelepípeda en piedra local, en cuya cara superior todavía es visible el hueco para albergar el pie izquierdo de la estatua dedicada al homenajeado, que se encontraría en los baños termales de época imperial, que ocupaba el lugar del antiguo gimnasio (como

\footnotetext{
${ }^{16}$ Brennan 2000: 481 señala que esta pudo ser la ocasión en que Norbano volvió a Roma, pero no hay nada seguro.
} 
parece probar el descubrimiento en las inmediaciones de la base honoraria IG XIV 616 = I.Rhegion 5) (Münzer 1932: 234, Di Giacomo 2010: 145). El texto del epígrafe

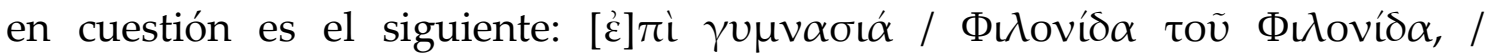

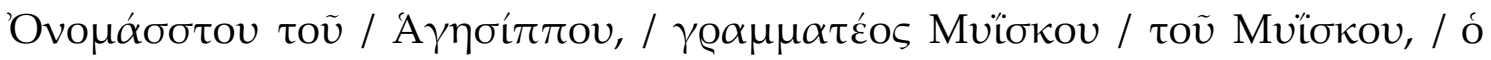

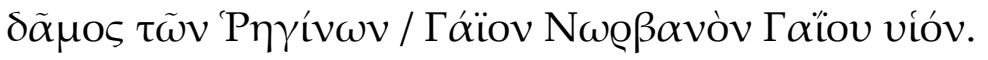

En un principio, esta inscripción se podría fechar en la segunda mitad del siglo I a.C., basado esencialmente en consideraciones generales. Ante todo, en la observación de Estrabón de que en su tiempo en la antigua Magna Grecia, a excepción de Neapolis, Tarentum y Rhegium, todas las comunidades griegas habían sido barbarizadas, por lo que eran las únicas que habían conservado su carácter griego (Str. 6, 1, 2) (Putorti 1927: 7, Münzer 1932: 234).

Un análisis del epígrafe por G. di Giacomo revela que ésta fue dedicada al hijo del pretor, debido principalmente a la falta de mención alguna a su cargo o a cualquier título honorífico. Posiblemente, como acontece en la gala Lugdunum, en donde se documentan dos bases para L. Dasumius Tullius Tuscus (CIL VI 1526) y su hijo M. Dasumius Tullius Varro (CIL VI 1400), para este autor había un epígrafe

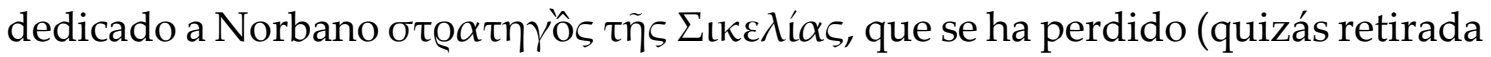
en su momento tras la victoria de Sila en la Guerra Civil), y otra a su hijo antes de ser triumvir monetalis (Di Giacomo 2010: 167-181).

El caso de Norbano (que según G. Manganaro tendría una relación de patronato con Rhegium) (Manganaro 1989: 179 n. 61) trae a colación un hecho que muchas veces ha pasado desapercibido: la actuación del gobernador

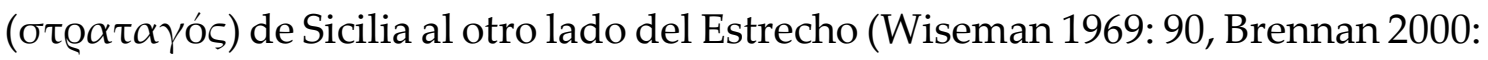
836 n. 32, Prag 2007b: 234, Díaz Fernández 2017: 82). A este respecto, se puede mencionar, junto con Norbano, al anónimo pretor del Lapis Pollae (AE $1956149=$

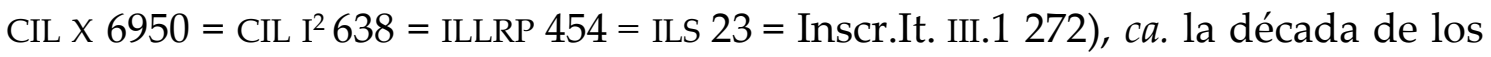
años 130s a.C.; a Cn. Aufidio T. f. (IG XIV 612 = I.Rhegion $1=$ SIG $^{3}$ 715), ca. el año 100 a.C., al que ya hemos aludido; a C. Verres (pr. 74 a.C.), gobernador de la isla durante los años 71-73 a.C., quien solicitó madera precisamente a los habitantes de Rhegium (Cic. 2Verr. 5, 18, 47); y a M. Porcio Catón (pr. 54 a.C.), en el año 49 a.C., quien envió legados a Lucania y el Bruttium para efectuar levas de ciudadanos romanos mientras era gobernador de Sicilia (Caes. BCiv. 1, 30, 4). De esto parece deducirse que una de las tareas de los administradores de la isla era la vigilancia de los Estrechos, al menos, el de Mesina, como parece atestiguar las fuentes anteriores.

\section{BIBLIOGRAFÍA}

Amela VAlverde, L. (2007), El toro contra la loba. La guerra de los Aliados (91-87 a.C.), Madrid, Signifer Libros.

Amela VAlverde, L. (2020), «La campaña de Pompeyo en Sicilia (82 a.C.)», Myrtia 35, 225-248. 
ANDREAU, J. (2007), «Registers, account-books, and written documents in the de Frumento», en Sicilia nutrix plebis romanae. Rhetoric, Law, and Taxation in Cicero's Verrines, Prag, J. R. W. (ed.), London, Institute of Classical Studies, 81-92.

BABElon, E. (1886), Description Historique et Chronologique des Monnaies de la République Romaine vulgairement appelés monnaies consulaires. Tome Deuxième, Paris, Rollin et Feuardent.

BADIAN, E. (1964), «Notes on Provincial Governors from the Social War down to Sulla's Victory», en Studies in Greek and Roman History, Oxford, Blackwell, 71-104.

BADIAN, E. (1962), «Waiting for Sulla», JRS 52, 47-61.

BADIAN, E. (1983), «The Silence of Norbanus», AJPh 104, 160-164.

BARLOW, Ch. T. (1980), «The Roman Government and the Roman Economy, 92-80 B.C.», AJPh 101, 202-219.

BRENNAN, T. C. (2000), The Praetorship in the Roman Republic. Volume II, Oxford, Oxford University Press.

BRoughton, T. R. S. (1952), The Magistrates of the Roman Republic. Volume 2. 99 B.C.-31 B.C, New York, American Philological Association.

Broughton, T. R. S. (1986), The Magistrates of the Roman Republic. Volume 3. Supplement, Atlanta, Scholar Press.

CAMPAGNA, L. (2007), «Architettura pubblica ed evergetismo nella Sicilia di età republicana», en La Sicilia romana tra repubblica ed alto impero. Atti del convengo di studi, Miccichè, E., Moldeo, S. y Santogan. L. (a cura de), Caltanissetta, SiciliAntica, 110-134.

CARNEY, T. F. (1961), «The Flight and Exile of Marius», GER 8, 98-121

CRAWFORD, M. H. (1974), Roman Republican Coinage. 2 vols, Cambridge, Cambridge University Press.

DART, CHR. J. (2014), The Social War, 91 to 88 BCE. A History of the Italian Insurgency against the Roman Republic, London-New York, Routledge.

Di GIACOMO, G. (2010), «Ancora sulla base onoraria di C. Norbanus (S.E.G. I 418)», en Giornata di studi per Lidio Gasperini, Arnaldi, A., Lanzillotta, E. y Antolini, S. (a cura de), Roma, Edizioni Tored, 143-182.

Di PAOLA, L. (1999), «Le vie di communicazione», en Magna Grecia e Sicilia. Stato degli studi e prospettive di ricerca, Barra Bagnasco, M., De Miro, E. y Pinzone, A. (eds.), Messina, Università di Messina, 459-469.

DÍAZ ARIÑO, B. (2015), Miliarios romanos de época republicana, Roma, Quasar.

DÍAZ FERNÁNDEZ, A. (2015), Provincia et Imperivm. El mundo provincial en la República romana (227-44 a.C.), Sevilla, Editorial Universidad de Sevilla.

DíAZ FERNÁNDEZ, A. (2017), "A Survey of the Roman Provincial Command from Republican Epigraphy, The Cases of Sicily and Sardinia», en Insularity, Identity and Epigraphy in the Roman World, Velaza, J. (ed.), Newcastle upon Tyne, Cambridge Scholars Publishing, 69-92.

Dubouloz, J. y PitTIA, S. (2009), «La Sicile romaine, de la disparition du royaume de Hiéron II à la réorganisation augustéenne des Provinces», Pallas 80, 85-125.

Evans, R. (1987), «Norbani Flacci, The Consuls of 38 and 24 BC», Historia 36, 121-128.

Evans, R. J. (1995), Gaius Marius. A Political Biography, University of South Africa, Pretoria. 
Grueber, H. A. (1910), Coins of the Roman Republic in the British Museum. Vol. I. Aes rude, aes signatum, aes grave, and coinage of Rome from B.C. 268, London, Yje British Museum.

GRuen, E. (1968), Roman Politics and the Criminal Courts, 149-78 B.C., Cambridge, Harcard University Press.

Holm, A. (1965), Storia della Sicilia nell' Antichità III, Roma, Forni.

JASHEMSKI, W. F. (1950), The Origins and History of the Proconsular and the Propraetorian Imperium to 27 B.C., Chicago, The University of Chicago Press.

JeNisOn, E. S. (1919), The History of the Province of Sicily, Boston, The Colonial Press.

Kendall, S. (2013), The Struggle for Roman Citizenship. Romans, Allies, and the Wars of 9177 BCE, Piscatawaym, Gorgias Press.

KONRAD, C. F. (1997), «Marius at Eryx (Sallust, P. Rylands 473. 1)», Historia 46, 26-83.

LAST, H. A. Y GARDNER, M. A. (1932), «The enfranchisement of Italy», en The Cambridge Ancient History. Volume IX. The Roman Republic 133-44 B.C., Cook, S. A., Adcock, F. E. y Charlesworth, M. A. (eds.), Cambridge, University of Cambridge Press, 158210.

LONG, G. (1866), The Decline of the Roman Republic. Volume II, London, Bell \& Dadly.

MANGANARO, G. (1972), «Per una Storia della Sicilia Romana», ANRW I 1, 442-461.

MANGANARO, G. (1989), «Iscrizioni latine nuove e vecchie della Sicilia», Epigraphica 51, 161-209.

MANGANARO, G. (2012), Pace e guerra nella Sicilia tardo-ellenistica e romana (215 a.C.-14 d.C.), Ricerche storiche e numismatiche, Bonn, Habelt.

MAtsubara, T. (1998), Diodorus Siculus on the Late Roman Republic, Diss. Edinburgh.

MerimeE, P. (1841), Essai sur La Guerre Sociale, Paris, Typographie de Firmin Didot Freres.

MiccichÈ, C. $\left(2020^{2}\right)$, L'isola più vella. La Sicilia nella "Biblioteca Storica" di Diodoro Siculo, Caltanisseta, Lussografica.

Mommsen, TH. (1870), Histoire de la monnaie romaine. Tome second, Paris, Tollin et Feuardet.

MÜNZER, F. (1932), «Norbanus», Hermes 67, 220-236.

PAOlETTI, M. (1994), "Occupazione romana e storia delle città», en Storia della Calabria antica, 2. Età itálica e romana, Roma, Salvatore Settis, 465-556.

PinA POlO, F. y DíAZ FERnÁNDEZ, A. (2019), The Quaestorship in the Roman Republic, Berlin-Boston, De Gruyter.

PITTIA, S. (2012), «Diodore et l'histoire de la Sicilie républicaine», en Diodore d'Agyrion et l'Histoire de la Sicile. Collin-Bouffier, S. (ed.), Besançon, Presses Universitaires de Franche-Comté, 171-226.

PRAG, J. R. W. (2007a), «Auxilia and Gymnasia: A Sicilian Model of Roman Imperialism», JRS 97, 68-100.

PRAG, J. R. W (2007b), «Ciceronian Sicily: the Epigraphic Dimensions», en La Sicile de Cicéron. Lectures des Verrines, Dubouloz, J. y Pottia, S. (eds.), Besançon, Presses Universitaires de Franche-Comté, 245-271.

PRAG, J. R. W. (2007c), «Roman Magistrates in Sicily, 227-49 BC», en La Sicile de Cicéron. Regards croisés sur les Verrines, Dubouloz, J. y Pottia, S. (eds.), Besançon, Presses Universitaires de Franche-Comté, 287-310.

PRAG, J. R. W. (2010), «Troops and commanders: auxilia externa under the Roman Republic», Hormos 2, 101-113.

PutORTI, N. (1927), «Due iscrizioni di Reggio Calabria», Mouseion 1, 3-9. 
RODRÍGUEZ GONZÁLEZ, J. (2005), Diccionario de batallas de la Historia de Roma (753 a.C.-476 d.C.) [3.386 batallas libradas por los ejércitos romanos], Madrid, Signifer Libros.

SALMON, E. T. (1967), Samnium and the Samnires, Cambridge, Cambridge University Press.

SAMPSON, G. C. (2013), The Collapse of Rome. Marius, Sulla $\mathcal{E}$ the $1^{\text {st }}$ Civil War (91-70 BC), Barnsley, Pen and Sword.

SIRENA, G. (2007), «La viabilità costiera della Sicilia orientale in età romana: la cosidetta Via Pompeia», en La Sicilia romana tra repubblica ed alto impero. Atti del convengo di studi, Miccichè, E., Moldeo, S. y Santogan. L. (a cura de), Caltanissetta, SiciliAntica, 91-109.

SiRenA, G. (2011), Via Pompeia. L'antico tracciato stradale tra Messina e Siracusa, Roma, Bonanno.

SORACI, C. (2016), La Sicilia romana. Secc. III a.C.-V d.C., Roma, Carocci.

UGGERI, G. (2007), «La formazione del sistema stradale romano in Sicilia», en La Sicilia romana tra repubblica ed alto impero. Atti del convengo di studi, Miccichè, E., Moldeo, S. y Santogan. L. (a cura de), Caltanissetta, SiciliAntica, 228-243.

WALtON, F. R. (1967), Diodorus Siculus XII. Books XXXIII-XL. Index. With a English Translation by..., Cambridge-London, Harvard University Press.

WiLSON, R. J. A. (2013a), «Becoming Roman Overseas? Sicily and Sardinia in the Late Roman Republic», en A Companion to the Archaeology of the Roman Republic, Evans, J. D. (dir.), Malden-Oxford-Chichester, Wiley-Blackwell, 485-504.

WILSON, W. J. A. (2013), «Hellenistic Sicily, c. 270-100 BC», en The Hellenistic West. Rethinking the Ancient Mediterranean, Prag, R. W. y Quinn, J. C. (eds.), Cambridge, Cambridge University Press, 79-119.

WISEMAN, T. P. (1969), «Viae Anniae Again», PBSR 37, 82-91.

WISEMAN, T. P. (1971), New Men in the Roman Senate 139 BC-AD 14, Oxford, Oxford University Press. 